\title{
ON THE FRICTIONAL CONTACT IN CRACK ANALYSIS
}

\author{
MARIA COMNINOU \\ Department of Applied Mechanics and Engineering Science, University of Michigan Ann Arbor, MI 48109 , \\ U.S.A. \\ and \\ J. DUNDURS \\ Department of Civil Engineering, Northwestern University, Evanston, II 60201, U.S.A
}

\begin{abstract}
Following the idea of Bowie and Freese we consider a crack in a field of pure bending. The crack is closed over part of its extent, and the two crack faces in contact are allowed to slip under frictional constraints. The solution is constructed under the assumption that the applied loading is monotonically increased. It is discovered, however, that it is possible to analyze unloading by suitably superposing the present solution and that of Bowie and Freese.
\end{abstract}

\section{INTRODUCTION}

BOWIE AND FREESE proposed in a recent paper[1] to correct certain crack solutions that yield overlapping of the crack faces by introducing closure without slip in a segment of the crack. Their solution is expected to be applicable for relatively large values of the coefficient of friction. For smaller values, slip occurs and the boundary conditions have to be modified accordingly. In this paper we extend the Bowie-Freese analysis to include the effect of friction. We use a singular integral equation approach rather than complex function techniques.

More specifically, the problem deals with a crack in a field of pure bending. We first investigate under what conditions the crack faces in the contact region will stick and when they will slip. Next we give the solution for frictional slip in the contact region under monotonically increasing loading. It is discovered, however, that due to certain features of this solution it is possible to continue the analysis by superposition into the regime of decreasing loading which eventually leads to backslip.

\section{FORMULATION AND SOLUTION FOR A MONOTONICALLY INCREASED LOAD}

Consider a crack of length $L_{1}+L_{2}$ in a field of pure bending. The geometry and placement of the coordinate axes are shown in Fig. 1. We assume on the basis of the Bowie-Freese solution that the crack is open in the interval $-L_{1}<x<b$ and closed in the interval $b<x<L_{2}$, but that the contact zone consists of the slip zone $b<x<c$ and the stick zone $c<x<L_{2}$.

The bending field in the absence of the crack is taken as

$$
\bar{\sigma}_{x x}=m \bar{y}, \quad \bar{\sigma}_{x y}=\tilde{\sigma}_{y y}=0 .
$$

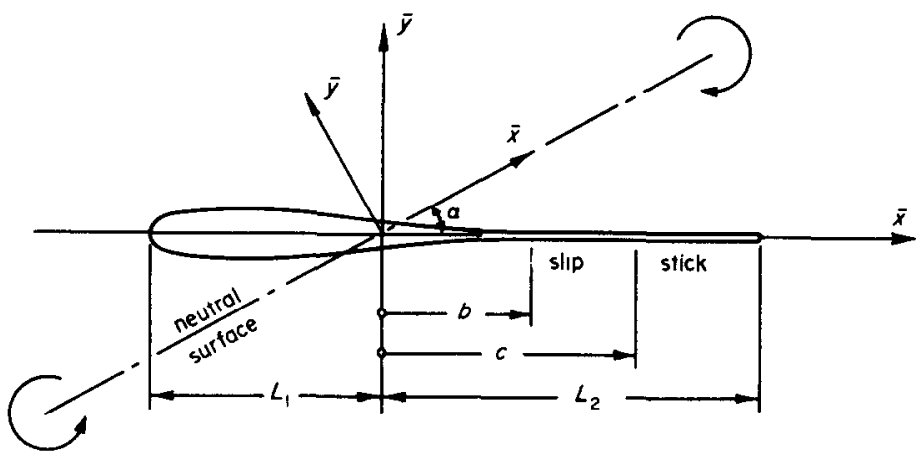

Fig. 1. Partially closed crack in a field of pure bending. 
At first, $m=m(t)$ is restricted to be a monotonically increasing function of time with $m(0)=0$. The tractions on $y=0$ corresponding to the bending field are

$$
\begin{aligned}
& \sigma_{x y}(x, 0)=-m x \cos \alpha \sin ^{2} \alpha \\
& \sigma_{y y}(x, 0)=-m x \sin ^{3} \alpha .
\end{aligned}
$$

We represent the crack as an array of distributed edge dislocations. It may be recalled [2] for this purpose that a discrete dislocation with the Burgers vector $\left(b_{x}, b_{y}\right)$ situated at $(\xi, 0)$ in an infinite medium induces the following stress components at $(x, 0)$ :

$$
\sigma_{x y}=-\frac{C b_{x}}{\pi} \frac{1}{\xi-x}, \quad \sigma_{y y}=-\frac{C b_{y}}{\pi} \frac{1}{\xi-x}
$$

where

$$
C=\frac{2 \mu}{\kappa+1}
$$

$\mu$ is the shear modulus, $\kappa=3-4 \nu$ in plane strain with $\nu$ being Poisson's ratio. Denoting by $B_{x}(x)$ and $B_{y}(x)$ the densities of the distributed dislocations, we note that

$$
B_{x}(x)=-\frac{\mathrm{d} h(x)}{\mathrm{d} x}, \quad B_{y}(x)=-\frac{\mathrm{d} g(x)}{\mathrm{d} x}
$$

where

$$
h(x)=u_{x}\left(x, 0^{+}\right)-u_{x}\left(x, 0^{-}\right)
$$

is the tangential shift of the upper crack face with respect to the lower, and

$$
g(x)=u_{y}\left(x, 0^{+}\right)-u_{y}\left(x, 0^{-}\right)
$$

is the gap between the crack faces. Consequently, $B_{x}(x)$ vanishes in $c<x<L_{2}$ and $B_{y}(x)$ in $b<x<L_{2}$.

On the basis of (4), the boundary conditions give immediately the integral equations for the dislocation densities.

Vanishing normal tractions on $-L_{1}<x<b$ :

$$
N(x)=-m x \sin ^{3} \alpha-\frac{C}{\pi} \int_{-L_{1}}^{b} \frac{B_{y}(\xi)}{\xi-x} \mathrm{~d} \xi=0 .
$$

Vanishing shearing tractions on $-L_{1}<x<b$ :

$$
S(x)=-m x \cos \alpha \sin ^{2} \alpha-\frac{C}{\pi} \int_{-L_{1}}^{c} \frac{B_{x}(\xi)}{\xi-x} \mathrm{~d} \xi=0
$$

Coulomb law of friction in the slip zone $b<x<c$ :

$$
S(x)=f N(x)
$$

or

$$
-m x \cos \alpha \sin ^{2} \alpha-\frac{C}{\pi} \int_{-L_{1}}^{c} \frac{B_{x}(\xi)}{\xi-x} \mathrm{~d} \xi=f\left\{-m x \sin ^{3} \alpha-\frac{C}{\pi} \int_{-L_{1}}^{b} \frac{B_{y}(\xi)}{\xi-x} \mathrm{~d} \xi\right\}
$$

where $f$ is the coefficient of friction. Condition (12) is written by anticipating from (2) that the upper crack face slips to the left with respect to the lower face. 
In addition, certain auxiliary conditions must be satisfied.

Single valued displacements:

$$
\int_{-L_{1}}^{c} B_{x}(\xi) \mathrm{d} \xi=0, \quad \int_{-L_{t}}^{b} B_{y}(\xi) \mathrm{d} \xi=0
$$

Non-negative gap or no overlapping of material:

$$
g(x) \geq 0, \quad-L_{1}<x<b .
$$

Compressive normal tractions in the slip and stick zones:

$$
N(x) \leq 0, \quad b<x<L_{2} .
$$

Condition of no slip in the stick zone:

$$
|S(x)|<f|N(x)|, \quad c<x<L_{2} .
$$

Consistency between directions of slip and shearing tractions:

$$
\operatorname{sgn} S(x)=\operatorname{sgn} \frac{\mathrm{d} h(x)}{\mathrm{d} t}, \quad b<x<c .
$$

It is clear that $B_{y}(x)$ and $b$ can be determined solely from (10) and (15). Consequently, the extent of the open part of the crack, the crack opening displacement, the normal tractions on $y=0$ and, thus, the Mode I stress intensity factor at $-L_{1}$ are not affected by the conditions in the closed segment of the crack. We merely record the corresponding results for future reference since they are essentially the same as in[1]. Using the new variables

$$
\begin{array}{cc}
x=\delta \zeta+\sigma, & \xi=\delta \omega+\sigma \\
\delta=\frac{1}{2}\left(b+L_{1}\right), & \sigma=\frac{1}{2}\left(b-L_{1}\right)
\end{array}
$$

which normalize the integral equation, $B_{y}$ solved from (10) is

$$
B_{y}(\zeta)=\frac{m}{C} \sin ^{3} \alpha(\delta \zeta+b)(1-\zeta)^{1 / 2}(1+\zeta)^{-1 / 2}, \quad-1<\zeta<1
$$

and it follows from (15) that

$$
b=\frac{1}{3} L_{1}
$$

Thus the extent of the open part of the crack does not depend on the level of the applied loads. The normal tractions from (10) and (22) are then

$$
N(\zeta)=-m \sin ^{3} \alpha\left\{\sigma|\zeta-1|^{1 / 2}|\zeta+1|^{-1 / 2}+\delta \operatorname{sgn} \zeta\left(\zeta^{2}-1\right)^{1 / 2}\right\}, \quad|\zeta|>1
$$

and the stress intensity factor in tension follows as

$$
K_{1}\left(-L_{1}\right)=m \sin ^{3} \alpha\left(2 L_{1} / 3\right)^{3 / 2} .
$$

To examine the range of validity of the Bowie-Freese analysis we assume first that there is no slip zone, or that $c=b$. The solution of (11) with $c$ replaced by $b$ is obtained by following Muskhelishvili[3] and is similar to that of (10):

$$
B_{x}(\zeta)=\frac{m}{C} \cos \alpha \sin ^{2} \alpha(\delta \zeta+b)(1-\zeta)^{1 / 2}(1+\zeta)^{-1 / 2}
$$


which satisfies condition (14). The shearing tractions outside the open part of the crack are

$$
S(\zeta)=-m \cos \alpha \sin ^{2} \alpha\left\{\sigma|\zeta-1|^{1 / 2}|\zeta+1|^{-1 / 2}+\delta \operatorname{sgn} \zeta\left(\zeta^{2}-1\right)^{1 / 2}\right\}, \quad|\zeta|>1 .
$$

Condition (18) now yields

$$
f>\cot \alpha .
$$

Thus closure without slip is possible and the Bowie-Freese analysis remains valid as long as (28) is satisfied. The stress intensity factor in shear is then from (27)

$$
K_{2}\left(-L_{1}\right)=m \cos \alpha \sin ^{2} \alpha\left(2 L_{1} / 3\right)^{3 / 2} .
$$

Since the functional forms of $N(\zeta)$ and $S(\zeta)$ from (24) and (27) are the same, it is expected that, for lower values of $f$ than required by (28), the slip zone will extend to $L_{2}$. In view of (10), eqns (11) and (13) can be combined into the single integral equation

$\frac{C}{\pi} \int_{-L_{1}}^{L_{2}} \frac{B_{x}(\xi)}{\xi-x} \mathrm{~d} \xi=-m x \cos \alpha \sin ^{2} \alpha+f\left\{m x \sin ^{3} \alpha+\frac{C}{\pi} \int_{-L_{1}}^{b} \frac{B_{y}(\xi)}{\xi-x} \mathrm{~d} \xi\right\}, \quad-L_{1}<x<L_{2}$.

Using $B_{y}$ from (22) in the original variables, and substituting into (30), we obtain

$$
\begin{aligned}
\frac{C}{\pi} \int_{-L_{1}}^{L_{2}} \frac{B_{x}(\xi)}{\xi-x} \mathrm{~d} \xi= & -m x \cos \alpha \sin ^{3} \alpha+f m \sin ^{3} \alpha\left\{\left(x+L_{1}\right)^{1 / 2}(x-b)^{1 / 2}\right. \\
& \left.\left.+\sigma(x-b)^{1 / 2}\left(x+L_{1}\right)^{-1 / 2}\right\} H(x-b)-H\left(x-L_{2}\right)\right\}, \quad-L_{1}<x<L_{2} .
\end{aligned}
$$

Normalizing the integration interval by setting

$$
\begin{array}{cc}
x=\delta^{\prime} s+\sigma^{\prime}, & \zeta=\delta^{\prime} r+\sigma^{\prime} \\
\delta^{\prime}=\frac{1}{2}\left(L_{2}+L_{1}\right), & \sigma^{\prime}=\frac{1}{2}\left(L_{2}-L_{1}\right)
\end{array}
$$

(31) becomes

$$
\begin{aligned}
\frac{C}{\pi} \int_{-1}^{1} \frac{B_{x}(r)}{r-s} \mathrm{~d} r= & -m \cos \alpha \sin ^{2} \alpha\left(\delta^{\prime} r+\sigma^{\prime}\right)+f m \sin ^{3} \alpha\left\{\left(\delta^{\prime} s+\delta^{\prime}\right)^{1 / 2}\left(\delta^{\prime} s+\delta^{\prime}-b\right)^{1 / 2}\right. \\
& +\sigma\left(\delta^{\prime} s+\sigma^{\prime}-b\right)^{1 / 2}\left(\delta^{\prime} s+\delta^{\prime}\right)^{-1 / 2}\left\{H\left(\delta^{\prime} s+\sigma^{\prime}-b\right)-H\left(\delta^{\prime} s-\delta^{\prime}\right)\right\}, \quad-1<s<1 .
\end{aligned}
$$

Since $x=L_{2}$ is the transition point from a slip to an adhesion zone, $B_{x}(x)$ has a square root singularity at $L_{2}$ [4]. Thus the solution of (34) is from [3]

$$
\begin{aligned}
B_{x}(s)= & \frac{m}{C} \cos \alpha \sin ^{2} \alpha\left(\frac{1}{2} \delta^{\prime}-\sigma^{\prime} s-\delta^{\prime} s^{2}\right)\left(1-s^{2}\right)^{-1 / 2} \\
& -\frac{f m}{\pi C} \sin ^{3} \alpha\left(1-s^{2}\right)^{-1 / 2} \int_{\left(b-\sigma^{\prime}\right) / \delta^{\prime}}^{1}\left[\left(\delta^{\prime} r+\delta^{\prime}\right)^{1 / 2}\left(\delta^{\prime} r+\delta^{\prime}-b\right)^{1 / 2}\right. \\
& \left.+\sigma\left(\delta^{\prime} r+\sigma^{\prime}-b\right)^{1 / 2}\left(\delta^{\prime} r+\delta^{\prime}\right)^{-1 / 2}\right] \frac{\left(1-r^{2}\right)^{1 / 2} \mathrm{~d} r}{r-s}+D\left(1-s^{2}\right)^{-1 / 2}
\end{aligned}
$$

Applying (14) on (35) we find that the constant $D$ must vanish.

Equation (35) essentially completes the solution for slip in the contact zone. It remains to be verified, however, that the appropriate auxiliary conditions are satisfied, since they were not incorporated in the solution procedure. Conditions (16) and (17) are readily seen to be satisfied for $m \geq 0$ and $0 \leq \alpha \leq \pi / 2$ in view of (22) and (24). Condition (19) is less straightforward to 
verify. From (12) and (24) it follows that $S(x) \leq 0$ in the slip zone for $m \geq 0$ and $0 \leq \alpha \leq \pi / 2$. The tangential shift $h(x)$ must be computed numerically from (7) using (35). The results of the calculations showed that (19) is indeed satisfied for $\mathrm{d} m(t) / \mathrm{d} t>0$.

The stress intensity factors in shear can be expressed in terms of $B_{x}(x)$ as

$$
\begin{gathered}
K_{2}\left(-L_{1}\right)=-C \lim _{x \rightarrow-L_{1}}\left\{\left(L_{1}+x\right)^{1 / 2}\left(L_{2}-x\right)^{1 / 2} B_{x}(x)\right\} \\
K_{2}\left(L_{2}\right)=C \lim _{x \rightarrow L_{2}}\left\{\left(L_{1}+x\right)^{1 / 2}\left(L_{2}-x\right)^{1 / 2} B_{x}(x)\right\} .
\end{gathered}
$$

The normalized stress intensity factors

$$
k_{2}()=\frac{K_{2}()}{m\left[\frac{1}{2}\left(L_{1}+L_{2}\right)\right]^{3 / 2} \cos \alpha \sin ^{2} \alpha}
$$

are shown vs $L_{2} / L_{1}$ for different values of $f \tan \alpha$ in Fig. 2.

\section{DECREASING LOAD AND BACKSLIP}

The normal tractions are the same for either stick or slip in the contact zone. Thus from (24)

$$
N(x)=-m(t) F(x) \sin ^{3} \alpha, \quad b<x<L_{2}
$$

where

$$
F(x)=(x-b)^{1 / 2}\left\{\sigma\left(x+L_{1}\right)^{-1 / 2}+\left(x+L_{1}\right)^{1 / 2}\right\}, \quad b>0, b<x<L_{2} .
$$

The shearing tractions are from (27)

$$
S^{B}(x)=-m(t) F(x) \cos \alpha \sin ^{2} \alpha, \quad b<x<L_{2}
$$

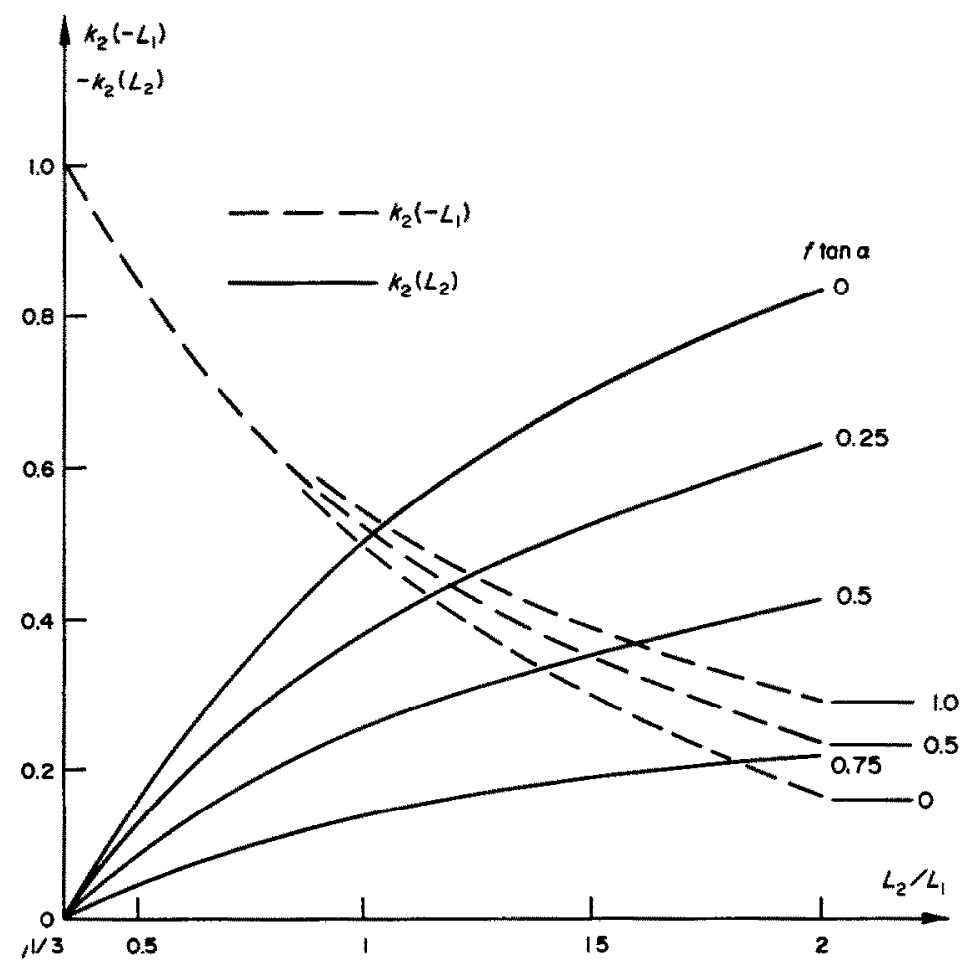

Fig. 2. Shear stress intensity factors. 


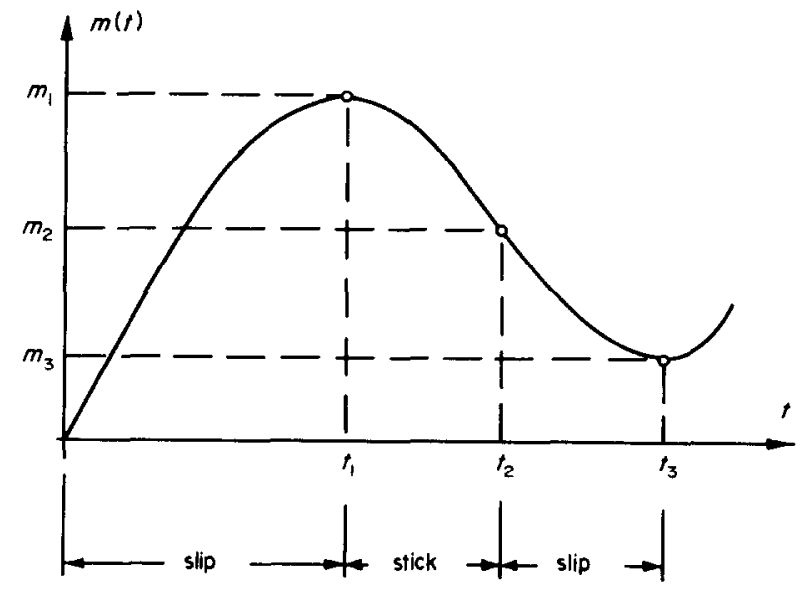

Fig. 3. Loading function leading to locking and backslip.

for stick (case of $f>\cot \alpha$ ), and from (12)

$$
S^{\mathcal{C}}(x)=-f m(t) F(x) \sin ^{3} \alpha, \quad b<x<L_{2}
$$

for slip (case of $f<\cot \alpha$ ) in the contact zone upon monotonically increased loading. Due to the fact that $N(x), S^{B}(x)$ and $S^{C}(x)$ are of the same functional form in $x$, it is possible to analyze unloading and the ensuing backslip, or for that matter to treat any course of loading specified by $m(t)$.

Let us consider a load function $m(t)$ of the form shown in Fig. 3 and adopt the notation

$$
m(t)= \begin{cases}m_{I}(t), & 0<t<t_{1} \\ m_{1}-m_{I I}(t), & t_{1}<t<t_{3}\end{cases}
$$

where $m_{1}=m\left(t_{1}\right)$, and $m_{I I}(t)$ is a monotonically increasing function. In the interval $0<t<t_{1}$ the load function is monotonically increasing, and slip occurs for $f<\cot \alpha$. Equations (39) and (42) then are applicable with $m(t)$ replaced with $m_{I}(t)$. When $m(t)$ reaches its local maximum $m_{1}$ at $t=t_{1},(19)$ is violated. Consequently the crack faces must stick, and the stresses existing at this instant are locked in as residual stresses. We assume that stick prevails from $t_{1}$ to some unknow time $t_{2}$. The additional tractions then are given by (39) and (41) with $m(t)$ replaced by $-m_{I I}(t)$. Thus the total tractions in the contact zone are

$$
\begin{aligned}
N(x) & =\left\{-m_{1}+m_{I I}(t)\right\} F(x) \sin ^{3} \alpha, \quad b<x<L_{2} \\
S(x) & =\left\{-f m_{1} \sin \alpha+m_{I I}(t) \cos \alpha\right\} F(x) \sin ^{2} \alpha, \quad b<x<L_{2} .
\end{aligned}
$$

The stress intensity factors for the unloading phase can be obtained by a similar superposition of the previous results. Stick in the contact zone lasts as long as (18) is not violated. Substituting (44) and (45) into (18) we get

$$
m_{2}=m\left(t_{2}\right)=\frac{\cot \alpha-f}{\cot \alpha+f} m_{1}
$$

at which backslip starts.

The analysis can be continued along the same lines in the backslip region and also beyond time $t_{3}$. Since the results can be deduced from those given so far, we refrain from going into the details. 


\section{REFERENCES}

[1] O. L. Bowie and C. E. Freese, On the "overlapping" problem in crack analysis. Engng Fracture Mech. 8, 373-379 (1976).

[2] J. Weertman and J. R. Weertman, Elementary Dislocation Theory. Macmillan, New York (1964).

[3] N. I. Muskhelishvili, Singular Integral Equations. Noordhoff, Leyden (1953).

[4] J. Dundurs and M. Comninou, Some consequences of the inequality conditions in contact and crack problems. $J$. Elasticity, 9, (1979)

(Received 18 July 1978; received for publication 14 November 1978) 